\title{
"The Sticky LAD": Large Intracoronary Thrombus in an Aneurysmal Left Anterior Descending Artery Presenting as ST-Elevation Myocardial Infarction
}

\author{
Jaime Alfonso M. Aherrera ${ }^{\mathrm{a}}$, Marc Denver A. Tiongson ${ }^{\mathrm{a}, \mathrm{b}}$, Eric Oliver D. Sison ${ }^{\mathrm{a}}$
}

\begin{abstract}
Management of acute coronary syndrome in coronary ectasia and large intracoronary thrombi has been culled from case reports. A 55-year-old male sought consult for severe chest pain. On angiography, there was note of large burden intracoronary thrombus. Various percutaneous coronary techniques were done. Angiographic success was not achieved, and then he was maintained on tirofiban. Repeat angiography after 2 weeks revealed dissolution of thrombus with optimal medical therapy. Management of atypical cases should be individualized.
\end{abstract}

Keywords: Intracoronary thrombus; Coronary artery ectasia; Coronary artery aneurysm

\section{Introduction}

Diffuse coronary aneurysm or ectasia is a relatively uncommon finding on angiography. The management of acute coronary syndrome (ACS) in patients with coronary ectasia and large intracoronary thrombi has been culled from case reports. Due to the large coronary segments and heavy thrombus burden, standard treatment may fail. Distal microembolization and improper stent sizing among these patients also raised concern during early percutaneous coronary intervention (PCI) in ACS [1]. We present a case of a patient with diffuse coronary ectasia and a large intracoronary thrombus presenting with STelevation myocardial infarction (STEMI). He was managed successfully with triple antiplatelets (aspirin, clopidogrel, and intracoronary and intravenous GIIb/IIIa inhibitors), anticoagulation, and adjunctive PCI (thrombectomy, angioplasty, and stenting).

Manuscript submitted May 30, 2018, accepted June 20, 2018

aSection of Cardiology, University of the Philippines, Philippine General Hospital, Manila, Philippines

${ }^{b}$ Corresponding Author: Marc Denver A. Tiongson, Section of Cardiology, University of the Philippines, Philippine General Hospital, Manila, Philippines. Email: denver_tiongson@yahoo.com

doi: https://doi.org/10.14740/jmc3101w

\section{Case Report}

A.P., a 55-year-old male, non-diabetic, non-hypertensive, sought consult for severe chest pain. He had no prior complaints, until 2 - $3 \mathrm{~h}$ prior to admission; he experienced sudden onset chest pain at rest, VAS 10/10, retrosternal and heavy in character, and associated with diaphoresis and dyspnea. He sought consult at the UP-PGH emergency room. He is a 30-pack-year smoker, but denies alcohol or illicit drug use. He had a history of dengue fever during childhood. He has no history of hypertension, diabetes, stroke, peripheral vascular disease, or renal disease. His father was hypertensive and his older brother died of "heart disease". He had no other complaints in other systems.

At the emergency room, he was received with on-going chest pain. Vital signs were as follows: blood pressure 90/60 $\mathrm{mm} \mathrm{Hg}$, heart rate 90 beats per minute, respiratory rate 26 per minute. He had no neck vein engorgement, bibasal rales, and no wheezing. His apex beat was not displaced. S1 was soft. $\mathrm{He}$ had a normal rate and regular rhythm and no murmurs. An electrocardiogram revealed sinus rhythm, normal axis, complete right bundle branch block, and ST-elevation in leads V1-V6, I, and aVL (with reciprocal changes in II, III, aVF), consistent with a massive ST-elevation myocardial infarction. Troponin-I was significantly elevated. Other blood workups were non-contributory. Primary percutaneous coronary intervention (PCI) was offered, however, the patient opted medical management. He was successfully thrombolysed immediately with streptokinase 1.5 million units intravenously. Postthrombolysis, ST-segment elevations decreased in amplitude, chest pain resolved, and vital signs remained stable. He was then transferred to the medical ICU on oral medications. We planned to do coronary angiography with intent to revascularize should the patient consent during the admission.

On the third hospital day, he had recurrence of angina, mild (VAS 2/10) in severity and heavy in character. We emphasized the importance of rescue PCI, for which he then consented. He was immediately brought up to the cath lab. Coronary angiography revealed a right dominant circulation with a dilated (ectatic) right coronary artery, left anterior descending (LAD), and left circumflex artery. Vessel diameters ranged from 5.2 - $5.9 \mathrm{~mm}$. The LMCA, RCA, and LCX were free of significant disease. In the proximal LAD, there was note of a round, lucent structure measuring $5.3 \times 5.8 \mathrm{~mm}$ and almost totally obstructing flow to the distal segments. This was consistent 

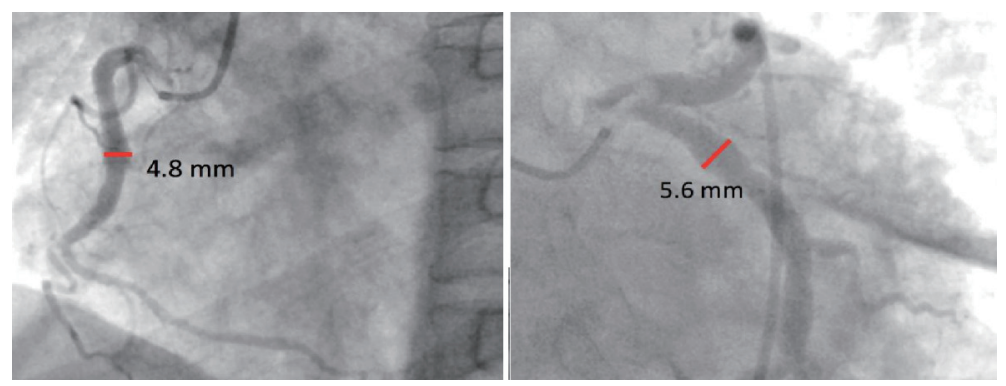

Figure 1. Images above show an ectatic right coronary artery and left circumflex, free of significant disease.
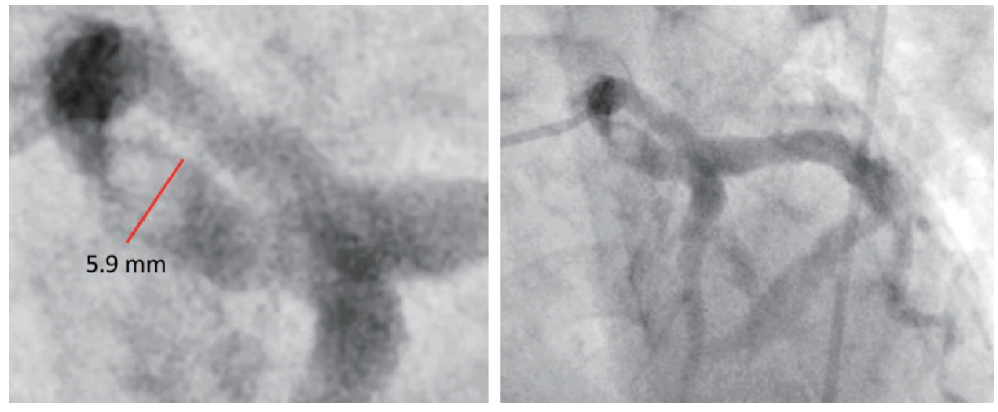

Figure 2. Images above show an ectatic left anterior descending coronary artery with note of a large, round, lucent structure in the proximal LAD, consistent with an intracoronary thrombus.

with a large burden intracoronary thrombus. The figures (Fig. $1,2)$ demonstrate the angiographic images.

He was managed with intravenous heparin, oral and intravenous antiplatelets, and various percutaneous techniques. A PTCA balloon was inserted and inflated at the area of the thrombus. Placement of a drug eluting stent was planned, but a large-sized stent was not available. Manual thrombectomy was also done with multiple passes, but resolution of the thrombus was not achieved. Final angiogram showed reduced obstruction in the proximal LAD, but still with TIMI flow 2 with the large thrombus still present. Intracoronary and intravenous tirofiban was then given, with intent to repeat the coronary angiogram between 1 - 2 weeks. The procedure was uneventful. Repeat angiogram 2 weeks after revealed dissolution of the thrombus with triple antiplatelets (aspirin, clopidogrel, tirofiban, and hep- arin). Figure 3 shows the final angiographic shots.

\section{Discussion}

Detection of intracoronary thrombi heralds a poor prognosis in STEMI [1]. Effects of intracoronary thrombus impair epicardial flow by occlusion of major coronary vessels and impair perfusion of the myocardium by distal embolization. Intracoronary thrombus is angiographically defined as the presence of a filling defect with either: 1) Total occlusion with convex, irregular, or hazy distal margins and post-injection contrast retention of staining; and 2) Partial occlusion circumferentially outlined by contrast medium [1]. Thrombus may be graded (TG) by the TIMI thrombus grading. TIMI TG 0 - 3 suggests small thrombus

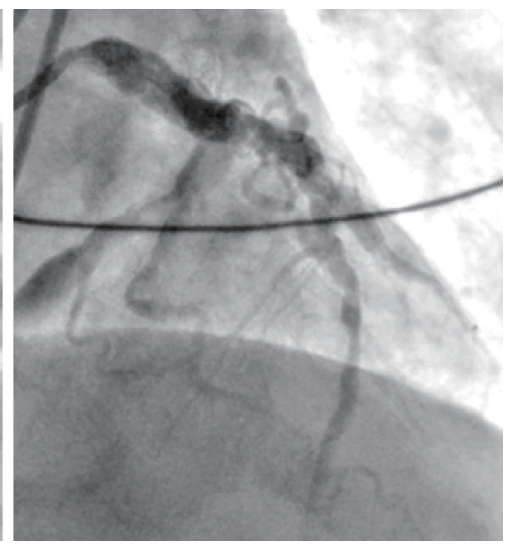

Figure 3. Repeat angiogram after 2 weeks shows resolution of the intracoronary thrombus. 
Table 1. Cases Published Involving Intracoronary Thrombus

\begin{tabular}{llll}
\hline Author & General data & Angiogram & Management \\
\hline Kuhl et al [5] & 75-year-old male, NSTE-ACS & $\begin{array}{l}\text { RCA: occluded with distal } \\
\text { thrombus }\end{array}$ & $\begin{array}{l}\text { Medical: nitrates, heparin, aspirin, clopidogrel, } \\
\text { long-term warfarin; PCI with stenting of the RCA }\end{array}$ \\
Lee et al [6] & 46-year-old male, STEMI & $\begin{array}{l}\text { RCA: totally occluded with } \\
\text { large thrombus burden }\end{array}$ & $\begin{array}{l}\text { Medical: eptifibatide, heparin, aspirin, clopidogrel, } \\
\text { long-term warfarin; Thrombectomy; No stenting done }\end{array}$ \\
Varghese et al [7] & 22-year-old male, NSTE-ACS & $\begin{array}{l}\text { LAD: ectasia with thrombus } \\
\text { in proximal LAD }\end{array}$ & $\begin{array}{l}\text { Medical: eptifibatide, heparin, aspirin, clopidogrel, } \\
\text { dabigatran; Aspiration thrombectomy and stenting }\end{array}$ \\
\hline
\end{tabular}

burden; while TIMI TG 4 suggests a large thrombus burden [2].

Management of STEMI is straightforward, and immediate reperfusion is necessary. Subgroups may benefit more from adjunctive pharmacological and interventional strategies, such as large thrombus burden. In our case, aside from PCI with stenting of the involved segment, thrombectomy may be of benefit for angiographic success $[3,4]$, which was done. The combination of STEMI with ectatic (aneurysmal) coronaries and a large intracoronary thrombus burden is uncommon and its management varies from a few published case reports. The common denominator in the management of the few similar cases documented is adjunctive therapeutic decisions, including thrombectomy and GIIb-IIIa-inhibitors. Table 1 [5-7] summarized published case reports with similar presentation and management.

\section{Conclusions}

We have demonstrated that thrombectomy, angioplasty, chronic anticoagulation, and aggressive antiplatelet therapy (given orally, intracoronary, and intravenously) may be effective in these cases. Management of such atypical and rare cases should be individualized. Controlled studies are necessary.

\section{References}

1. Vecchio S, Varani E, Chechi T, Balducelli M, Vecchi G,
Aquilina M, Ricci Lucchi G, et al. Coronary thrombus in patients undergoing primary PCI for STEMI: Prognostic significance and management. World J Cardiol. 2014;6(6):381-392.

2. Gibson CM, de Lemos JA, Murphy SA, Marble SJ, McCabe CH, Cannon CP, Antman EM, et al. Combination therapy with abciximab reduces angiographically evident thrombus in acute myocardial infarction: a TIMI 14 substudy. Circulation. 2001;103(21):2550-2554.

3. Henriques JP, Zijlstra F, Ottervanger JP, de Boer MJ, van 't Hof AW, Hoorntje JC, Suryapranata H. Incidence and clinical significance of distal embolization during primary angioplasty for acute myocardial infarction. Eur Heart J. 2002;23(14):1112-1117.

4. Rezkalla SH, Kloner RA. Coronary no-reflow phenomenon: from the experimental laboratory to the cardiac catheterization laboratory. Catheter Cardiovasc Interv. 2008;72(7):950-957.

5. Kuhl M, Varma C. A case of acute coronary thrombosis in diffuse coronary artery ectasia. J Invasive Cardiol. 2008;20(1):E23-25.

6. Lee HH, Lin TH, Su HM, Voon WC, Lai WT, Sheu $\mathrm{SH}$, Hsu PC. Recurrent thrombosis in a case of coronary ectasia with large thrombus burden successfully treated by adjunctive warfarin therapy. Acta Cardiol Sin. 2013;29(5):462-466.

7. Varghese, et al. Rare case of CAE presenting with Thrombus Embolization and AMI. 2011. 